\title{
Lithium Quantum Consciousness
}

\author{
Angus McCoss \\ Kilbrandon House, Scotland, UK \\ Email: ammcc0@outlook.com
}

How to cite this paper: McCoss, A. (2017) Lithium Quantum Consciousness. Journal of Quantum Information Science, 7, 125-139. https://doi.org/10.4236/jqis.2017.74010

Received: October 4, 2017

Accepted: November 5, 2017

Published: November 8, 2017

Copyright $\odot 2017$ by author and Scientific Research Publishing Inc. This work is licensed under the Creative Commons Attribution International License (CC BY 4.0).

http://creativecommons.org/licenses/by/4.0/

\begin{abstract}
Conscious agency is considered to be founded upon a quantum state of mind $|\Xi\rangle$. An original synthesis, called "Lithium Quantum Consciousness" (LQC), proposes that this quantum state utilises lithium-6 (spin-1) qutrit nuclear magnetic resonance (NMR) quantum information processing (QIP) in the connectome (brain-graph). In parallel to the connectome's processing of physiological controls, perception, cognition and intelligence via quantum electrodynamics (QED), the connectome also functions via its dynamic algebraic topology as a unitary transceiver antenna laced with lithium- 6 nuclei which are spin-entangled with each other and with the environmental vortical gluon field via quantum chromodynamics (QCD). This unitary antenna (connectome) bestows the self its unity of consciousness within an intertwined-history multi-agent environment. An equivalence is proposed between Whitehead's occasions of experience and topological spacetime instantons in the vortical gluon field. Topological spacetime instantons pervade the vortical gluon field in a quantum information network of vortex interactions, herein termed the "instanton-net", or "Instanet" [sic]. The fermionic isotope lithium-6 has a very low nuclear binding energy and the smallest non-zero nuclear electric quadrupole moment of any stable nucleus making it susceptible to quantum chromodynamic (QCD) interaction with the vortical gluon field and ideal for spin-1 qutrit NMR-QIP. The compact spherical atomic orbital of lithium provides ideal rotational freedom inside tetrahedral water cages in organo ${ }^{6} \mathrm{Li}^{+}\left(\mathrm{H}_{2} \mathrm{O}\right)_{4}$ within which the lithium nucleus rapidly tumbles for NMR motional narrowing and long decoherence times. Nuclear spin-entanglement, among water-caged lithium- 6 nuclei in the connectome, is a spin-1 qutrit NMR-QIP resource for conscious agency. By contrast, similar tetrahedral xenon cages in organo ${ }^{6} \mathrm{Li}^{+} \mathrm{Xe}_{4}$ excimers are postulated to decohere the connectome's NMR-QIP due to xenon's NMR signal being extremely sensitive to its molecular environment. By way of this quantum neurochemistry, lithium is an effective psychiatric medication for enhancing mood and xenon is an effective anaesthetic.
\end{abstract}




\section{Keywords}

Instanton, Qutrit, Artificial Intelligence, Quantum Chromodynamics, Connectome

\section{Introduction}

This paper offers a new and original synthesis, called Lithium Quantum Consciousness (LQC), which comes about through the extension of the author's research programme on quantum intelligent cosmology, into the realm of quantum conscious cosmology. A metaheuristic which outlines a qutrit-based quantum deep-learning Triuniverse concept [1] and a paper which proposes that the entangled agency of life accounts for dark matter phenomena [2] together provide some context and preamble for this present paper.

A key theme and claim which carries through my programme into this paper is Nature's proposed fundamental, evolutionary and multi-levelled deep-learning quantum ternary computation by way of qutrits, not qubits. Another continued theme is that the naturally entangled conscious agency of life and its evolution seems pivotal to observed physical phenomena, from the Planck to cosmological scales.

Furthermore, a meta-stable Reality seems to exist despite profound uncertainties in its quantum information foundations. We shall see in the course of this paper that quantum field fluctuations and their associated instantons (networked vortical field configurations with a "topological twist") form a quantum information computation network (Figure 1) with which the quantum state of mind $|\Xi\rangle$ is connected.
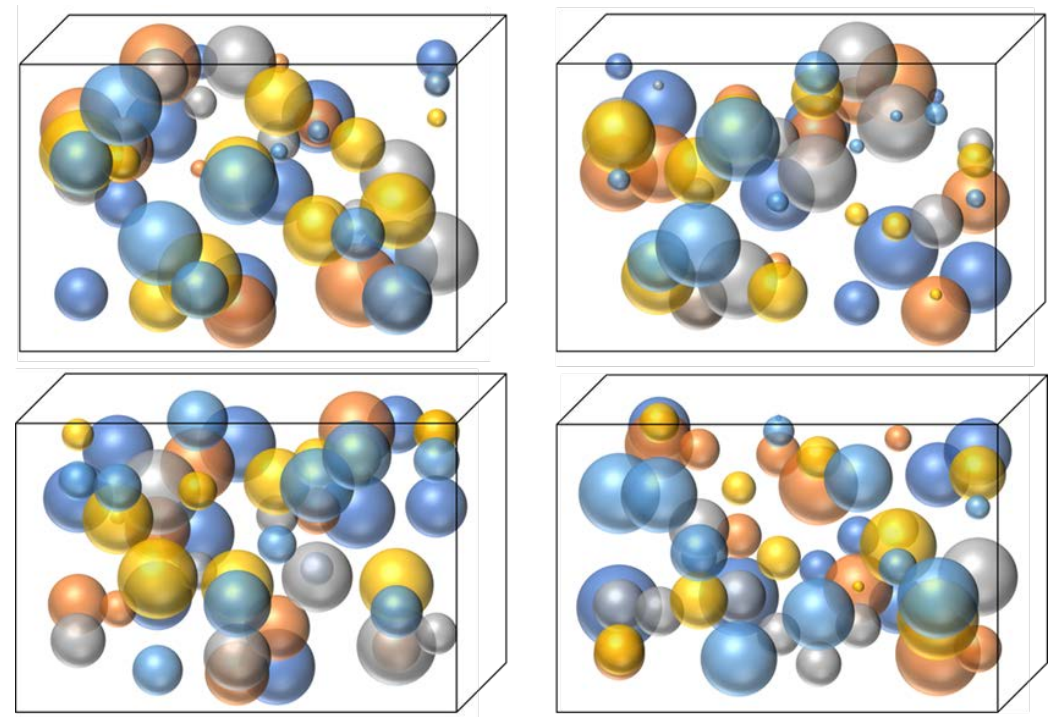

Scale: Edges few $10^{-15} \mathrm{~m}$. Simulation speed $10^{24}$ frames $\mathrm{s}^{-1}$. Four random frames.

Figure 1. The gluon field is a dynamical function of spacetime. Four schematic snapshots provide an impression of the energetically evolving topological charge density structure of gluon-field fluctuations sketched from lattice QCD visualisations. Fleeting instantons correlate with this dynamic structure. 


\section{LQC Synthesis, Sources and Hypotheses}

Seminal works on the quantum state of mind by Fisher [3] [4] [5] and Penrose and Hameroff [6] [7] [8] provided stimulating catalysts for extending my research from quantum intelligent cosmology to quantum conscious cosmology. The reader is also directed to their extensive review bibliographies.

Fisher has been developing ideas on nuclear spin quantum information processing as an explanation for the workings of the conscious mind. He highlights the long established positive effects that lithium has on those suffering from mood disorders [9] [10] and he draws our attention to laboratory rat experiments by Sechzer et al. [11] who showed that the stable isotopes of lithium (lithium-6 and lithium-7) have different effects on the rats' minds. It seems lithium-6 differentially elevates cognitive activity, which was an unexpected result given the close electrochemical characteristics of the isotopes. Furthermore, a human being in a magnetic field of 8 Tesla may experience vertigo, nausea, metallic taste sensations and perceptions of light flashes [12]. Fisher [5] has since embarked on a quest to reverse engineer quantum cognition, examining the possibility of processing with nuclear spins in the brain [3].

In framing this quest, Fisher constrains his search to find a common biological element (CHNOPS: carbon, hydrogen, nitrogen, oxygen, phosphorus and sulfur) with an isolated nuclear spin, to serve as a "neural qubit", i.e. an atom with a nuclear spin $1 / 2$. I believe this qubit 2-level quantum system constraint on the search leads to a conclusion which is not necessarily the most natural. Nonetheless, by applying that 2-level constraint, Fisher rationally concludes that phosphorous-31 is the sought after "neural qubit" and he operationalises it by screening it from rapid decoherence within Posner molecules, $\mathrm{Ca}_{9}\left(\mathrm{PO}_{4}\right)_{6}$.

Alternatively, consistent with my quantum intelligent cosmology programme and the quantum deep learning Triuniverse metaheuristic within it [1], I carry out a similar reverse engineering search but explore qutrit 3-level quantum systems instead.

A qutrit has three orthogonal basis states $|-1\rangle,|0\rangle$, and $|1\rangle$ which are used to describe the balanced ternary quantum state of mind $|\Xi\rangle$ as a superposition in the form of a linear combination of the three states:

$$
|\Xi\rangle=c_{1}|-1\rangle+c_{2}|0\rangle+c_{3}|1\rangle
$$

where the coefficients $c_{1}, c_{2}$ and $c_{3}$ are probability amplitudes, such that the sum of their squares is unity:

$$
\left|c_{1}\right|^{2}+\left|c_{2}\right|^{2}+\left|c_{3}\right|^{2}=1
$$

A qutrit is argued to be the smallest system that exhibits inherent quantum features such as contextuality [13], which has been conjectured to be a resource for quantum computing [14] [15] [16]. The reader is referred to the bibliography in [1] relating to the natural effectiveness of qutrit and balanced ternary computing, see also [17].

We therefore seek a natural "neural qutrit", i.e. an atom with a nuclear spin-1 
and that turns out to be an acutely focused search criteria. In Nature, there are only three spin-1 stable light isotopes: hydrogen-2 (deuterium), lithium-6 and nitrogen-14. A textbook on spin-1 nuclear magnetic resonance (NMR), focuses on these three isotopes and has been written by Chandrakumar [18] —readers are directed to this for background and a bibliography. Amongst these three isotopes we can immediately identify a strong candidate for the sought after "neural qutrit", the isotope with a long established ability to alter the conscious mind [11], i.e. lithium-6.

Dogra, Dorai and Arvind [19] present an important recent study of the Majorana geometrical representation for a qutrit and use it to describe the action of quantum gates. Their work is supported by experiments on a spin-1 NMR qutrit system oriented in a liquid crystalline environment (spin-1 deuterium nucleus of a chloroform-D molecule). They also show that experimental implementation of these ternary quantum gates is validated by complete quantum state tomography which they carry out using Gell Mann matrices. I extend their mathematical and experimental treatment to lithium- 6 and propose that lithium- 6 is naturally isolated from short timescale decoherence when caged in water molecules and thereby is a naturally efficient qutrit computation resource for the quantum mind. On the third candidate spin-1 isotope, nitrogen-14, it does have potential as a qutrit computation resource (e.g. nitrogen-vacancy centres in diamond) and I refer the reader to selected work on qutrit computing [20] [21].

Solvation of lithium in water clusters is described amongst others by [22] [23] [24] [25] [26] and the structure where the lithium atom is surrounded by $n=4$ $\left(\mathrm{H}_{2} \mathrm{O}\right)_{\mathrm{n}}$ molecules in the first shell is found to be the most stable for both neutral and cationic $n \geq 4$ clusters. I propose the compact spherical atomic orbital of lithium provides ideal rotational freedom inside tetrahedral water cages (Figure 2 ) in organo ${ }^{6} \mathrm{Li}^{+}\left(\mathrm{H}_{2} \mathrm{O}\right)_{4}$ within which the lithium nucleus rapidly tumbles for NMR motional narrowing and long decoherence times. Nuclear spin-entanglement, among water-caged lithium-6 nuclei in the connectome (brain-graph), is thereby a spin-1 qutrit NMR-QIP resource for conscious agency.

A further characteristic of lithium-6 NMR is significant in this synthesis, namely

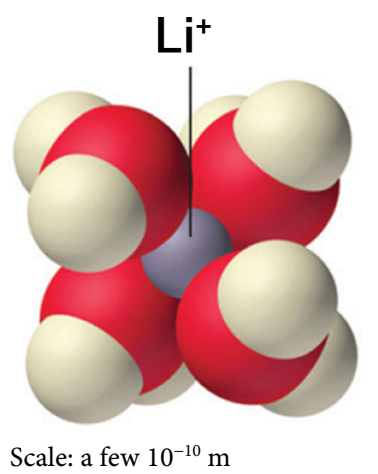

Figure 2. In aqueous solution, $\mathrm{Li}^{+}$forms the tetrahedral $\mathrm{Li}^{+}\left(\mathrm{H}_{2} \mathrm{O}\right)_{4}$ complex. Structure of $\mathrm{Li}^{+}\left(\mathrm{H}_{2} \mathrm{O}\right)_{4}$ : Lithium ${ }^{+}$(purple) in centre surrounded by a tetrahedral cage of four water molecules. Oxygen (red). Hydrogen (white). Image credit https://chem.libretexts.org. 
the very narrow natural linewidth of lithium-6 $(\Gamma / 2 \pi=5.87 \mathrm{MHz})$ [27]. This NMR spike response enables effective low-error signaling during QIP in the connectome.

Another key catalyst for my research into quantum conscious cosmology has been the collaborative works of Penrose and Hameroff [6] [7] [8]. Notwithstanding their ground-breaking studies of the potential function of microtubules in quantum neural processing, I have been particularly drawn to their echoing of the seminal metaphysical work of Alfred North Whitehead (1861-1947) in their discussions on "proto-conscious" events. Whitehead monumentally introduced a metaphysically primitive notion, which he called an "actual occasion". For him an "actual occasion" was a process of becoming, not an enduring substance and as Whitehead put it, "actual occasions" are the "final real things of which the world is made up", they are "drops of experience, complex and interdependent" [28]. I propose an equivalence between Whitehead's actual occasions of experience $\omega_{\varepsilon}$ and topological spacetime instantons $x\left(\tau_{\varepsilon}\right)$, where $\tau_{\varepsilon} \rightarrow 0$, in the vortical gluon field (Figure 1):

$$
\omega_{\varepsilon} \equiv x\left(\tau_{\varepsilon}\right)
$$

The instanton solution jumps from one vacuum $x=-1$ to another vacuum $x=1$ instantly where $\tau_{\varepsilon} \rightarrow 0$ hence the term "instanton". The spatiotemporal absence of an instanton, represented instantaneously by the empty vacuum, embodies a third and zero state, $x=0$, see Equation (1) and note spacetime states of instantons thus represent a qutrit resource. Equation (3) also relates to the constituents of what philosophers call qualia.

For background, studies and bibliographies on instantons the reader is referred to [29]-[38]. For example, the Yang Mills action describes the behaviour of gluons. A characteristic of the Yang-Mills action is that there are finite-action topological soliton solutions to the classical field equations. These solitons are instantons, or pseudoparticles in early papers [37] [38].

The dynamic algebraic-topology of the connectome (brain-graph) is a subject of active neuroscientific research [39] [40] [41]. It has motivated my research programme to relate ongoing connectome topology insights to the quantum topological foundations of quantum fields and cosmology [2].

Two particular characteristics of the physical connectome of white matter tracts of bundled myelinated axons are highlighted. Firstly, the trace element lithium is preferentially localised in the white matter tracts, rather than in the folded grey matter cortex [42] and I postulate lithium's arrangement within these wet fatty tracts includes the lithium-water complex $\mathrm{Li}^{+}\left(\mathrm{H}_{2} \mathrm{O}\right)_{4}$. Note also, anisotropic $\mathrm{H}_{2} \mathrm{O}$ diffusion in white matter in brain forms the basis for the utilization of Diffusion Tensor Imaging (DTI) to track the same fibre pathways (Figure 3) [43]. Secondly, using a continuous neural field model of excitatoryinhibitory interactions on the connectome, Atasoy et al. [44] demonstrate a neural mechanism behind the self-organization of connectome harmonics. Furthermore, they show the critical relation between a delicate excitation-inhibition 


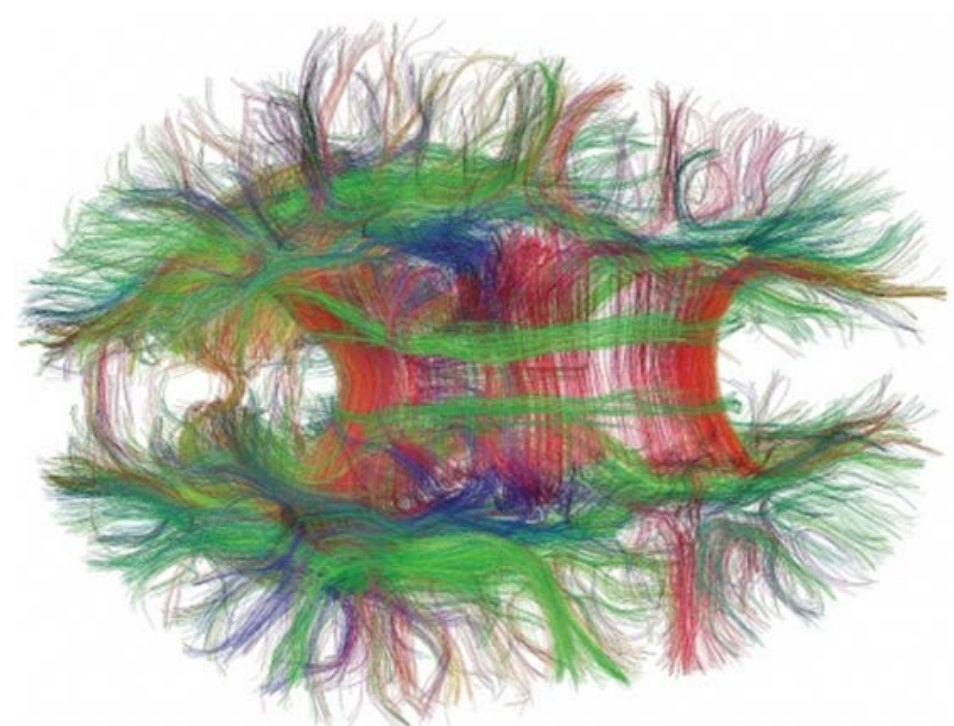

Scale: a few $10^{-1} \mathrm{~m}$ and gamma synchrony conscious moments of a few $10 \mathrm{~s}^{-1}[8]$.

Figure 3. Connectome (brain-graph) viewed from above, created with Diffusion Tensor Imaging (DTI), showing complex arrangement of fibrous white matter tracts. Image credit http://www3.imperial.ac.uk/icimages?p_imgid=515622.

balance and the neural field patterns matches neurophysiological changes observed during recovery and loss of consciousness. They provide examples of low frequency connectome harmonics, for given wave numbers and present corresponding spatial patterns of synchronous oscillations estimated by the eigenvectors of the connectome Laplacian.

Such neural field fluctuations are herein conjectured to correlate with low frequency quantum field fluctuation harmonics through a mediating role played by the connectome laced with entangled fermionic spin-1 lithium- 6 which provides the architecture for qutrit NMR-QIP. As such, this connectome architecture is an isotropic unitary transceiver antenna, or wireless router, for quantum information exchanges and for a deep-learning quantum information computer which hosts quantum consciousness. Whilst the connectome processes physiological controls, perception, cognition and intelligence via its quantum electrodynamics (QED), the author hypothesises that in parallel the connectome processes consciousness via quantum chromodynamics (QCD). Hyperfine interaction between the lithium-6 nucleus and its electron environment connects NMR-QIP of consciousness $(\mathrm{QCD})$ with the parallel processing of physiological controls, perception, cognition and intelligence (QED).

It is of note that colour confinement is a fundamental characteristic of QCD and this is conjectured herein to confine an individual's quantum state of mind $\Xi\rangle$ to the self and to prevent extra-cerebral telepathy. Had QED consciousness been naturally selected during biological evolution, instead of QCD consciousness, then perhaps the unconfined interactions of QED might have enabled quantum telepathy? It is the author's belief that such an unconfined ability would not necessarily bestow selective advantage. Indeed, unconfined quantum telepathy 
might be experienced by the conscious agent as a chaotic cacophony of multi-selves in a manner more debilitating than chronic schizophrenia. Hence NMR-QIP (QCD colour confined to the agent's own connectome) is Nature's selected physics underpinning the individual's privately confined experience of unity of consciousness.

Topological spacetime instantons pervade the vortical gluon field in a quantum information network of vortex interactions, herein termed the "instanton-net", or "Instanet" [sic]. The more familiar human invention which we know as the Internet (a global system of interconnected computer networks using common protocols) provides a convenient analogy for the postulated QCD "instanton-net", or "Instanet". In computer science terminology the QCD "Instanet" has an event-driven architecture, where an event is a change in state. Instantons in the gluon field are foundational to these events (Figure 4). Reality is experienced by a conscious agent via QCD connection through its connectome to the "Instanet of Things" which comprises interacting objects, intelligent devices (including lifeforms) and fellow conscious agents (sentient animals). Again we may look to the manmade Internet of Things for a convenient analogy. Lithium Quantum Consciousness (LQC) intersects the vortical gluon field in spacetime and quantum information entanglements connect consciousness with the "Instanet of Things".

Physical phenomena emerge from the Instanet-connected automation protocol, otherwise known as the (fundamentally tripartite [1]) Standard Model of particle physics. The known laws of physics govern the "Instanet". Each conscious agent and every lifeless thing is uniquely identifiable in the "Instanet of Things" through its embedded quantum information computations. Through

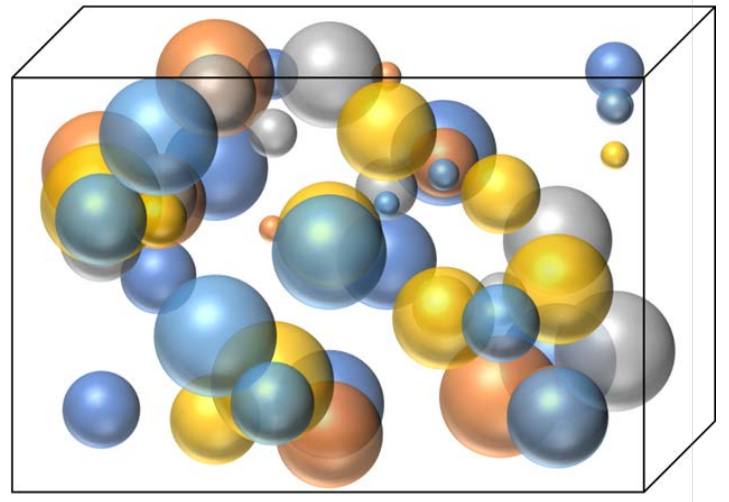

Scale: Edges few $10^{-15} \mathrm{~m}$. Simulation speed $10^{24}$ frames $\mathrm{s}^{-1}$. Single random frame.

Figure 4. Spacetime instantons pervade the vortical gluon field in a quantum information network of vortex interactions, herein termed the "instanton-net", or "Instanet" [sic]. In this schematic illustration (similar to Figure 1) consider the golden yellow orbs as correlating with instantons in the connectome's NMR-QIP ${ }^{6} \mathrm{Li}^{+}\left(\mathrm{H}_{2} \mathrm{O}\right)_{4}$-laced white matter tracts, whereas the other coloured orbs correlate with instantons in the "Instanet of Things", i.e. the physical and multi-agent environment. In this way Lithium Quantum Consciousness (LQC) intersects the vortical gluon field in spacetime and quantum information entanglements connect consciousness with the "Instanet of Things". 
their QCD consciousness the agents experience and impose their free will upon the multi-agent and multi-thing environment, which is the "Instanet of Things". All agents and all things inter-operate within the quantum information network of the spacetime instanton gluon field, the "Instanet of Things". In this way quantum information is the conserved currency of all interactions.

Interconnection of embedded devices in the "Instanet of Things", including "smart objects" (intelligent and conscious life forms), enables pervasive "automated" interactions under the laws of physics and enables advanced complex quantum information processes, like a "smart grid" or "society" of history-intertwined conscious life forms. Dumb objects (e.g. rock fragments) are also embedded devices in the "Instanet of Things" however they lack conscious free will and are thus slaves to the "automated" interactions under the laws of physics (e.g. accreting into planets subject to plate tectonics and erosion etc.). In essence, order emerges amidst fundamental uncertainty due to the quantum information interconnections amongst everything: dumb things, smart things and conscious agents. Selforganisation of spontaneous order in complex systems is thus enabled by the "Instanet of Things" and conscious agents have evolved therein to exert free will on the system evolution, thereby contributing to its quantum deep-learning [1].

Furthermore, QCD entanglement of the observer's conscious self with the vortical gluon field causes the "Measurement Problem" [45]. In other words, the consciousness of the conscious agent is inextricably entangled with the "Instanet of Things" (Figure 4), which includes all things, including instruments and physical experiments. It is impossible for the conscious agent to disconnect from the "Instanet of Things", at least without foregoing the experience of conscious life. The conscious agency of life has inescapable consequences.

Penultimately, work on excimers of lithium within tetrahedral xenon cages ${ }^{6} \mathrm{Li}^{+} \mathrm{Xe}_{4}$ [46] [47] prompted me to include that research topic within this present synthesis. Xenon is an established anaesthetic [48] and whilst its operation might be classical it is atomic not a molecular therefore we may consider xenon in the same NMR-QIP picture. I consider it to be significant that xenon's NMR signal is extremely sensitive to its molecular environment [49] (due to the xenon atom's large and highly polarizable electron cloud) and propose that tetrahedral xenon cages in organo ${ }^{6} \mathrm{Li}^{+} \mathrm{Xe}_{4}$ excimers decohere the connectome's NMR-QIP causing loss of consciousness. Thus, by way of quantum neurochemistry, lithium is an effective psychiatric medication for enhancing mood whilst xenon is an effective anaesthetic.

Finally, heavy metal atom interaction with organo ${ }^{6} \mathrm{Li}^{+}\left(\mathrm{H}_{2} \mathrm{O}\right)_{4}$ NMR-QIP impairs brain function. The brain has however naturally evolved neuroprotective countermeasures. The meninges are three membranes which envelope the brain. The innermost one, which adheres to the brain, is the delicate and fluid-impermeable "pia mater". Detoxifying neuroprotective metallothionein binds heavy metals which concentrate in the pia mater. The pia mater is thus a barrier which effectively keeps heavy metals outside the brain and has two positive consequences in this synthe- 
sis. One is that lithium-6 within the connectome is able to host NMR-QIP without heavy metal interference. The other is that quantum physical containment by heavy metal enriched pia mater shields (via electronic spin-orbit interaction contribution to nuclear magnetic shielding) [50] [51] and further confines lithium-6 spin-1 qutrit NMR-QIP to the spacetime intersection of the connectome with the vortical gluon field (Figure 4). With ageing, progressively heavy metal enriched pia mater increasingly isolates the older quantum mind from the "Instanet of Things" and thus from Reality. This would compound bio-degenerative processes of ageing.

\section{Summary List of LQC Components}

A summary list of the components of Lithium Quantum Consciousness (LQC):

1) Conscious agency is founded upon a quantum state of mind $|\Xi\rangle$;

2) Connectome (brain-graph) processes physiological controls, perception, cognition and intelligence via quantum electrodynamics (QED);

3) In parallel, the connectome also processes the experience of consciousness via quantum chromodynamics (QCD);

4) QCD colour confinement confines individual's quantum state of mind $|\Xi\rangle$ to the self;

5) Topological spacetime instantons pervade the vortical gluon field in a quantum information network of vortex interactions, termed the "instanton-net", or "Instanet" [ sic];

6) In computer science the "Instanet" has an event-driven architecture, where an event is a change in state and instantons in the gluon field are foundational to these events;

7) Reality is experienced by a conscious agent via QCD connectome connection to the "Instanet of Things" which comprises interacting objects, intelligent devices (including lifeforms) and fellow conscious agents (sentient animals);

8) All agents and all things inter-operate within the quantum information network of the spacetime instanton gluon field, the "Instanet of Things";

9) Natural, and therein neural, quantum information processing (QIP) involves qutrit quantum ternary computation, in a Triuniverse [1];

10) Lithium-6 is susceptible to QCD interaction with the vortical gluon field (hence the "Instanet") due to its very low nuclear binding energy and the smallest non-zero nuclear electric quadrupole moment of any stable nucleus;

11) Lithium-6 is ideal for spin-1 qutrit nuclear magnetic resonance (NMR) QIP;

12) Compact spherical atomic orbital of lithium provides ideal rotational freedom inside tetrahedral water cages in organo ${ }^{6} \mathrm{Li}^{+}\left(\mathrm{H}_{2} \mathrm{O}\right)_{4}$;

13) Nuclear spin-entanglement, among water-caged lithium-6 nuclei, is a spin-1 qutrit NMR-QIP resource for conscious agency;

14) Narrow natural linewidth of lithium- $6(\Gamma / 2 \pi=5.87 \mathrm{MHz})$ enables low-error spike QIP in the connectome;

15) Hyperfine interaction between the lithium-6 nucleus and its electron en- 
vironment connects NMR-QIP of consciousness (QCD) with the parallel processing of physiological controls, perception, cognition and intelligence (QED);

16) Conscious agent's lithium-laced white matter tracts act as an isotropic unitary transceiver antenna, and wireless router, connected to the "Instanet of Things" (Reality);

17) White matter unitary antenna is laced with lithium-6 nuclei which are spin-entangled with each other and the vortical gluon field;

18) White matter unitary transceiver antenna is a quantum computer, and wireless router, and bestows the self its unity of consciousness;

19) QCD entanglement of observer's self with the vortical gluon field leads to the "Measurement Problem" and the conscious agent is inextricably entangled with the "Instanet of Things" which includes all instruments and experiments;

20) Neural field fluctuations correlate with quantum field fluctuations and their harmonics;

21) Whitehead's actual occasions of experience are equivalent to topological spacetime instantons in the vortical gluon field (event-driven "Instanet" architecture);

22) Tetrahedral xenon cages in organo ${ }^{6} \mathrm{Li}^{+} \mathrm{Xe}_{4}$ excimers decohere the connectome's NMR-QIP due to xenon's NMR signal being extremely sensitive to its molecular environment.

Considering this above synthesis holistically and being founded on quantum information, I propose the following synopsis. Meta-stable societal coexistence and coevolution, emerges with a semblance of order from fundamental uncertainty, as a complex quantum system where the conscious agent, other agents and the environment exert natural actions, reactions and free will (in the case of conscious agents) on each other, thus building correlations through qutrit quantum deep-learning, intertwined-histories and braided worldlines. This dynamic evolving ensemble manifests itself to a conscious agent as our Triuniverse [1] which is an "Instanet of Things". As Whitehead [28] said, actual occasions of experience are the "final real things of which the world is made up" and I propose these are equivalent to topological spacetime instantons in the vortical gluon field, with which our Lithium Quantum Consciousness (LQC) connects.

\section{Discussion of Applications and Technologies}

Biological receptors, processors and transceivers of physiological controls, perception, cognition, intelligence and consciousness are all subject to evolutionary natural selection, with advanced organisms having more capable and computationally advantageous sets of integrated sensory faculties. From this vantage point across the evolutionary fitness landscape, our species Homo sapiens is reasonably elevated, when compared to say Caenorhabditis elegans (roundworm). However, the author believes the present earthly biological level of evolution of quantum states of consciousness is unlikely to be at the maximum natural potential. This belief is an extension of universal non-anthropocentrism. More hum- 
bly, perhaps human consciousness is close to a local maximum on the evolutionary fitness landscape, but we may be currently limited to the biological "foothills" of a mountain range of conscious potential.

Indeed, the proposals herein can be technologically tested, harnessed, amplified and augmented, in manmade lithium-water synthetic brains for quantum deep-learning artificial consciousness. Such lithium-water synthetic brains could in principle surpass those which have evolved so far within the biology of our planet. Much as nuclear reactors are controlled by elements with high neutron capture cross-sections, e.g. silver, indium, boron and cadmium, the proposed level of consciousness of the lithium-water synthetic brain could be controlled by variably substituting water with anaesthetic xenon, which decoheres the connectome's NMR-QIP, with the creation of unconscious lithium-xenon excimers.

With appropriate in vivo clinical care and ethical controls, lithium-water synthetic brain material in impermeable vessels could be implanted within the brain, under the skull, under or exterior to the scalp, even applied topically to provide an additional proximal resource for one's own quantum mind. An ethical purpose would be to provide prosthetic medical care for patients with dissociative, bipolar and mood disorders, though for the mentally fit, augmented conscious enlightenment would also be accessible through such technologies. Lithium-water NMR-QIP technologies could also form the basis of a brain-computer interface.

Notwithstanding, lithium-water synthetic brain material could be assembled ex vivo into an artificial functionally dynamic algebraic topological connectome. That artificial connectome could exploit quantum deep-learning to evolve its design such that subsequently adapted versions, assembled robotically, could attain ever higher levels of consciousness. The levels to which such ex vivo synthetic quantum consciousness might rise are incomprehensible to present day humans. This therefore demands our utmost respect for unintended consequences and prior open debate of this technological opportunity and ethical (if not existential) threat.

\section{Conclusions}

An equivalence is proposed between Whitehead's occasions of experience and topological spacetime instantons in the vortical gluon field. The conscious agency of life is thereby considered to be founded upon a quantum state of mind $|\Xi\rangle$. Topological spacetime instantons pervade the vortical gluon field in a quantum information network of vortex interactions, termed the "instanton-net", or "Instanet" [sic].

The fermionic isotope lithium- 6 has a very low nuclear binding energy and the smallest non-zero nuclear electric quadrupole moment of any stable nucleus making it susceptible to quantum chromodynamic (QCD) interaction with the vortical gluon field and ideal for spin-1 qutrit nuclear magnetic resonance (NMR) quantum information processing. The compact spherical atomic orbital of lithium provides ideal rotational freedom inside tetrahedral water cages in organo ${ }^{6} \mathrm{Li}^{+}\left(\mathrm{H}_{2} \mathrm{O}\right)_{4}$ within 
which the lithium nucleus rapidly tumbles for NMR motional narrowing and long decoherence times. The wet fatty white matter tracts of the brain's connectome are considered to be laced with trace amounts of organo ${ }^{6} \mathrm{Li}^{+}\left(\mathrm{H}_{2} \mathrm{O}\right)_{4}$.

In parallel to the connectome's processing of physiological controls, perception, cognition and intelligence via quantum electrodynamics (QED), the connectome also functions via its dynamic algebraic topology as a unitary transceiver antenna laced with lithium-6 nuclei which are spin-entangled with each other and with the environmental vortical gluon field via quantum chromodynamics (QCD). Lithium Quantum Consciousness (LQC) in sentient agents thereby intersects the vortical gluon field in spacetime and quantum information entanglements connect consciousness with the "Instanet of Things". QCD entanglement of an observer's conscious self with the vortical gluon field thus leads to the "Measurement Problem".

Technologies which may be developed from these ideas include spin-1 qutrit organo ${ }^{6} \mathrm{Li}^{+}\left(\mathrm{H}_{2} \mathrm{O}\right)_{4}$ NMR quantum computers which 1) ex vivo, exhibit artificial intelligence and artificial consciousness, 2) in vivo, in an impermeable vessel placed in or proximal to the brain, augment human intelligence and human consciousness, and 3) provide a brain-computer interface.

\section{Acknowledgements}

This original research is self-funded and I thank my reviewers and editors for their valuable support.

\section{References}

[1] McCoss, A. (2016) Quantum Deep Learning Triuniverse. Journal of Quantum Information Science, 6, 223. https://doi.org/10.4236/jqis.2016.64015

[2] McCoss, A. (2017) Agency of Life, Entropic Gravity and Phenomena Attributed to “Dark Matter". Journal of Quantum Information Science, 7, 67. https://doi.org/10.4236/jqis.2017.72007

[3] Fisher, M.P. (2017) Are We Quantum Computers, or Merely Clever Robots? International Journal of Modern Physics B, 31, 1743001.

[4] Weingarten, C.P., Doraiswamy, P.M. and Fisher, M.P. (2016) A New Spin on Neural Processing: Quantum Cognition. Frontiers in Human Neuroscience, 10.

[5] Fisher, M.P. (2015) Quantum Cognition: The Possibility of Processing with Nuclear Spins in the Brain. Annals of Physics, 362, 593-602. https://doi.org/10.1016/j.aop.2015.08.020

[6] Hameroff, S.R. and Penrose, R. (1996) Conscious Events as Orchestrated Space-Time Selections. Journal of Consciousness Studies, 3, 36-53.

[7] Hameroff, S. and Penrose, R. (1996) Orchestrated Reduction of Quantum Coherence in Brain Microtubules: A Model for Consciousness. Mathematics and Computers in Simulation, 40, 453-480. https://doi.org/10.1016/0378-4754(96)80476-9

[8] Hameroff, S. and Penrose, R. (2014) Consciousness in the Universe: A Review of the 'Orch OR' Theory. Physics of Life Reviews, 11, 39-78. https://doi.org/10.1016/j.plrev.2013.08.002

[9] Cipriani, A., Pretty, H., Hawton, K. and Geddes, J.R. (2005) Lithium in the Preven- 
tion of Suicidal Behavior and All-Cause Mortality in Patients with Mood Disorders: A Systematic Review of Randomized Trials. American Journal of Psychiatry, 162, 1805-1819. https://doi.org/10.1176/appi.ajp.162.10.1805

[10] Smith, K.A. and Cipriani, A. (2017) Lithium and Suicide in Mood Disorders: Updated Meta-Review of the Scientific Literature. Bipolar Disorders. John Wiley \& Sons Ltd., New York.

[11] Sechzer, J.A., Lieberman, K.W., Alexander, G.J., Weidman, D. and Stokes, P.E. (1986) Aberrant Parenting and Delayed Offspring Development in Rats Exposed to Lithium. Biological Psychiatry, 21, 1258-1266.

[12] Chakeres, D.W. and De Vocht, F. (2005) Static Magnetic Field Effects on Human Subjects Related to Magnetic Resonance Imaging Systems. Progress in Biophysics and Molecular Biology, 87, 255-265.

https://doi.org/10.1016/j.pbiomolbio.2004.08.012

[13] Kurzyński, P. and Kaszlikowski, D. (2012) Contextuality of Almost All Qutrit States Can Be Revealed with Nine Observables. Physical Review A, 86, Article ID: 042125. https://doi.org/10.1103/PhysRevA.86.042125

[14] Ladd, T.D., Jelezko, F., Laflamme, R., Nakamura, Y., Monroe, C. and O’Brien, J.L. (2010) Quantum Computers. Nature, 464, 45-53.

[15] Dogra, S., Arvind and Dorai, K. (2014) Determining the Parity of a Permutation using an Experimental NMR Qutrit. Physics Letters A, 378, 3452-3456.

[16] Gedik, Z., Silva, I.A., Çakmak, B., Karpat, G., Vidoto, E.L.G., Soares-Pinto, D.O., Fanchini, F.F., et al. (2015) Computational Speed-Up with a Single Qudit. Scientific Reports, 5, Article No. 14671. https://doi.org/10.1038/srep14671

[17] Smania, M., Elhassan, A.M., Tavakoli, A. and Bourennane, M. (2016) Experimental Quantum Multiparty Communication Protocols. NPJ Quantum Information, 2, Article No. 16010. https://doi.org/10.1038/npjqi.2016.10

[18] Chandrakumar, N. (1996) Spin-1 NMR. Vol. 34, Springer-Verlag, Berlin and Heidelberg $\mathrm{GmbH} \& \mathrm{Co}$.

[19] Dogra, S., Dorai, K. and Arvind (2017) Majorana Representation, Qutrit Hilbert Space and NMR Implementation of Qutrit Gates.

[20] Li, B., Yu, Z.H. and Fei, S.M. (2013) Geometry of Quantum Computation with Qutrits. Scientific Reports, 3, Article No. 2594. https://doi.org/10.1038/srep02594

[21] Shauro, V.P., Pekhterev, D.I. and Zobov, V.E. (2007) A Comparative Analysis of Two Methods of Realizing Elementary Logic Operators for a Quantum Computer on Qutrits. Russian Physics Journal, 50, 566-573.

https://doi.org/10.1007/s11182-007-0084-6

[22] Feller, D., Glendening, E.D., Kendall, R.A. and Peterson, K.A. (1994) An Extended Basis Set ab initio Study of $\mathrm{Li}+(\mathrm{H} 2 \mathrm{O}) \mathrm{n}, \mathrm{n}=1-6$. The Journal of Chemical Physics, 100, 4981-4997. https://doi.org/10.1063/1.467217

[23] Wójcik, M.J., Mains, G.J. and Devlin, J.P. (1995) Theoretical Study of [Li (H2O) n]+ and $[\mathrm{K}(\mathrm{H} 2 \mathrm{O}) \mathrm{n}]+(\mathrm{n}=1-4)$ Complexes. International Journal of Quantum Chemistry, 53, 49-56. https://doi.org/10.1002/qua.560530108

[24] Hashimoto, K. and Kamimoto, T. (1998) Theoretical Study of Microscopic Solvation of Lithium in Water Clusters: Neutral and Cationic Li (H2O) n ( $\mathrm{n}=1-6$ and 8). Journal of the American Chemical Society, 120, 3560-3570. https://doi.org/10.1021/ja972726+

[25] Zeng, Z., Liu, C.W., Hou, G.L., Feng, G., Xu, H.G., Gao, Y.Q. and Zheng, W.J. (2015) Photoelectron Spectroscopy and ab initio Calculations of Li (H2O) n-and Cs 
(H2O) n-(n=1-6) Clusters. The Journal of Physical Chemistry A, 119, 2845-2856. https://doi.org/10.1021/jp512177j

[26] Lamoureux, G. and Roux, B. (2006) Absolute Hydration Free Energy Scale for Alkali and Halide Ions Established from Simulations with a Polarizable Force Field. The Journal of Physical Chemistry B, 110, 3308-3322. https://doi.org/10.1021/jp056043p

[27] Gehm, M.E. Properties of ${ }^{6} \mathrm{Li}$. http://www.physics.ncsu.edu/jet/techdocs/pdf/PropertiesOfLi.pdf

[28] Whitehead, A.N. (1929) Process and Reality. Macmillan, New York.

[29] Hanany, A. and Tong, D. (2003) Vortices, Instantons and Branes. Journal of High Energy Physics, 2003, 037. https://doi.org/10.1088/1126-6708/2003/07/037

[30] Tong, D. (2005) TASI Lectures on Solitons: Instantons, Monopoles, Vortices and Kinks. https://arxiv.org/pdf/hep-th/0509216.pdf

[31] Gross, D.J., Pisarski, R.D. and Yaffe, L.G. (1981) QCD and Instantons at Finite Temperature. Reviews of Modern Physics, 53, 43. https://doi.org/10.1103/RevModPhys.53.43

[32] Schäfer, T. and Shuryak, E.V. (1998) Instantons in QCD. Reviews of Modern Physics, 70, 323. https://doi.org/10.1103/RevModPhys.70.323

[33] Novikov, V.A., Shifman, M.A., Vainshtein, A.I. and Zakharov, V.I. (1999) ABC of Instantons. In: ITEP Lectures on Particle Physics and Field Theory, Volume 1, 201-299.

[34] Petreczky, P. (2012) Lattice QCD at Non-Zero Temperature. Journal of Physics G: Nuclear and Particle Physics, 39, Article ID: 093002. https://doi.org/10.1088/0954-3899/39/9/093002

[35] Alexandrou, C., de Forcrand, P. and D’Elia, M. (2000) The Role of Center Vortices in QCD. Nuclear Physics A, 663, 1031c-1034c.

[36] Trewartha, D., Kamleh, W. and Leinweber, D. (2015) Evidence That Centre Vortices Underpin Dynamical Chiral Symmetry Breaking in SU (3) Gauge Theory. Physics Letters $B, 747,373-377$.

[37] 't Hooft, G. (1976) Computation of the Quantum Effects Due to a Four-Dimensional Pseudoparticle. Physical Review D, 14, 3432-3450.

https://doi.org/10.1103/PhysRevD.14.3432

[38] Belavin, A.A., Polyakov, A.M., Schwartz, A.S. and Tyupkin, Y.S. (1975) Pseudoparticle Solutions of the Yang-Mills Equations. Physics Letters B, 59, 85-87.

[39] Reimann, M.W., Nolte, M., Scolamiero, M., Turner, K., Perin, R., Chindemi, G., Markram, H., et al. (2017) Cliques of Neurons Bound into Cavities Provide a Missing Link between Structure and Function. Frontiers in Computational Neuroscience, 11, 48. https://doi.org/10.3389/fncom.2017.00048

[40] Tozzi, A., Peters, J.F., Fingelkurts, A.A., Fingelkurts, A.A. and Marijuán, P.C. (2017) Topodynamics of Metastable Brains. Physics of Life Reviews, 21, 1-20.

[41] Gastner, M.T. and Ódor, G. (2016) The Topology of Large Open Connectome Networks for the Human Brain. Scientific Reports, 6, Article No. 27249. https://doi.org/10.1038/srep27249

[42] Lichtinger, J., Gernhäuser, R., Bauer, A., Bendel, M., Canella, L., Graw, M., Schöpfer, J., et al. (2013) Position Sensitive Measurement of Lithium Traces in Brain Tissue with Neutrons. Medical Physics, 40, Article ID: 023501. https://doi.org/10.1118/1.4774053

[43] Beaulieu, C. (2002) The Basis of Anisotropic Water Diffusion in the Nervous System-A Technical Review. NMR in Biomedicine, 15, 435-455. https://doi.org/10.1002/nbm.782 
[44] Atasoy, S., Donnelly, I. and Pearson, J. (2016) Human Brain Networks Function in Connectome-Specific Harmonic Waves. Nature Communications, 7, Article No. 10340. https://doi.org/10.1038/ncomms10340

[45] Leggett, A.J. (2005) The Quantum Measurement Problem. Science, 307, 871-872.

[46] Martyna, G., Cheng, C. and Klein, M.L. (1991) Electronic States and Dynamical Behavior of LiXe $\mathrm{n}$ and CsXe n Clusters. The Journal of Chemical Physics, 95, 1318-1336. https://doi.org/10.1063/1.461115

[47] Al-Ahmari, M., Saidi, S., Dhiflaoui, J., Hassen, F. and Berriche, H. (2015) Structure and Stability of the $\mathrm{Li}+$ Xen and LiXen Clusters. Journal of Cluster Science, 26, 913-924. https://doi.org/10.1007/s10876-014-0780-7

[48] Lachmann, B., Armbruster, S., Schairer, W., Landstra, M., Trouwborst, A., Van Daal, G.J. and Erdmann, W. (1990) Safety and Efficacy of Xenon in Routine Use as an Inhalational Anaesthetic. The Lancet, 335, 1413-1415.

[49] Morgado, P., Bonifácio, R., Martins, L.F. and Filipe, E.J. (2013) Probing the Structure of Liquids with 129Xe NMR Spectroscopy: n-Alkanes, Cycloalkanes, and Branched Alkanes. The Journal of Physical Chemistry B, 117, 9014-9024. https://doi.org/10.1021/jp4060507

[50] Vaara, J., Ruud, K. and Vahtras, O. (1999) Second- and Third-Order Spin-Orbit Contributions to Nuclear Shielding Tensors. The Journal of Chemical Physics, 111, 2900-2909. https://doi.org/10.1063/1.479572

[51] Vícha, J., Foroutan-Nejad, C., Pawlak, T., Munzarová, M.L., Straka, M. and Marek, R. (2015) Understanding the Electronic Factors Responsible for Ligand Spin-Orbit NMR Shielding in Transition-Metal Complexes. Journal of Chemical Theory and Computation, 11, 1509-1517. https://doi.org/10.1021/ct501089z 